\title{
Prevalencia de fibrilación auricular y características de la fibrilación auricular no valvular en la población general. Registro AFINVA
}

\author{
Vicente Mora-Llabata ${ }^{a, *}$, Daniela Dubois-Marqués ${ }^{a}$, Ildefonso Roldán-Torres ${ }^{a}$, \\ Conrado Mateu-Navarro ${ }^{b}$, Juan J. Sanz-García ${ }^{c}$, Victoria Moreno-Ballester ${ }^{d}$, \\ Salvadora Mira-Gimeno ${ }^{e}$ y Fernando Albiñana-Fernández ${ }^{f}$
}

\author{
a Departamento de Salud Valencia-Dr Peset, Servicio de Cardiología, Hospital Universitario Dr. Peset, Valencia, España \\ b Departamento de Salud Valencia-Dr Peset, Centro de Salud Padre Jofré, Valencia, España \\ c Departamento de Salud Valencia-Dr Peset, Centro de Salud Luis Oliag, Valencia, España \\ 'Departamento de Salud Valencia-Dr Peset, Centro de Salud Ruzafa, Valencia, España \\ e Departamento de Salud Valencia-Dr Peset, Centro de Salud Ingeniero Joaquín Benlloch, Valencia, España \\ f Departamento de Salud Valencia-Dr Peset, Centro de Salud Fuente San Luis, Valencia, España
}

Recibido el 28 de septiembre de 2015; aceptado el 22 de marzo de 2016

Disponible en Internet el 25 de mayo de 2016

\section{PALABRAS CLAVE}

Fibrilación auricular; Epidemiología;

Agentes antiarritmia; Anticoagulación;

Atención primaria

\begin{abstract}
Resumen
Fundamento y objetivos: La fibrilación auricular es la arritmia mantenida más común. El estudio pretende conocer la prevalencia de fibrilación auricular en la población general, y evaluar las características y el manejo de los casos con fibrilación auricular no valvular.

Métodos: Estudio transversal exploratorio en una muestra de la población de un Departamento de Salud $(n=30.024)$ de sujetos mayores de 18 años con diagnóstico electrocardiográfico de fibrilación auricular en la historia clínica electrónica de atención primaria. Se analizan características clínicas, manejo y tratamiento.

Resultados: De un total de 629 pacientes con diagnóstico de fibrilación auricular, lo que representa una prevalencia del 2,1\% (8,06\% en > 65 años), fueron seleccionados para el estudio 505 casos con fibrilación auricular no valvular. La edad media fue $77,4 \pm 10$ años, $55 \%$ mujeres. Se objetivó cardiopatía estructural en el $32 \%$ de casos e insuficiencia cardiaca en el $29,5 \%$. El $72 \%$ de pacientes recibía tratamiento anticoagulante, $60 \%$ con antivitamina $\mathrm{K}$ y $12 \%$ con anticoagulantes directos. Entre los primeros, solo el 53\% mantenía un tiempo en rango terapéutico $\geq$ $65 \%$ según el método de Rosendaal. La forma de presentación persistente-permanente fue más frecuente $(60,8 \%)$, seguida de la paroxística $(39,2 \%)$.
\end{abstract}

\footnotetext{
En representación de los investigadores del estudio AFINVA (Anexo).

* Autor para correspondencia.

Correo electrónico: vmoral@comv.es (V. Mora-Llabata).
} 
Conclusiones: La prevalencia de fibrilación auricular fue del 2,1\%, aumentando con la edad, con elevada proporción de cardiopatía concomitante. La proporción de pacientes anticoagulados y la calidad de la anticoagulación son deficientes, revelándose así la necesidad de mejor monitorización y mayor utilización de nuevos anticoagulantes directos en los casos indicados. (c) 2016 Sociedad Colombiana de Cardiología y Cirugía Cardiovascular. Publicado por Elsevier España, S.L.U. Este es un artículo Open Access bajo la licencia CC BY-NC-ND (http:// creativecommons.org/licenses/by-nc-nd/4.0/).

\section{KEYWORDS}

Atrial fibrillation; Epidemiology; Antiarrhythmia agents; Anticoagulation; Primary care

\section{Introducción}

La fibrilación auricular es la arritmia cardiaca sostenida más común y se asocia con una morbi-mortalidad elevada ${ }^{1}$. Estudios observacionales han proporcionado datos sobre características, perfil de riesgo, manejo y resultados clínicos de pacientes con fibrilación auricular ${ }^{2-6}$.

La complicación más grave de la fibrilación auricular es el ictus, tanto por el pronóstico vital a corto plazo como por sus secuelas invalidantes, con el consiguiente aumento del gasto sanitario.

Los antagonistas de la vitamina $\mathrm{K}$ han actuado como piedra angular de la anticoagulación en la prevención de ictus en la fibrilación auricular durante varias décadas. En los últimos años se han incorporado nuevos anticoagulantes orales inhibidores directos de la trombina o del factor $\mathrm{Xa}$, tan seguros y eficaces al menos como los antagonistas de la vitamina $\mathrm{K}^{7-10}$. Existen guías de manejo clínico basadas en la evidencia ${ }^{7,11,12}$ que abogan por el uso de puntuaciones de riesgo para identificar aquellos pacientes con riesgo trombótico o de sangrado. Sin embargo, los médicos son reacios a prescribir profilaxis con tratamiento anticoagulante en una amplia proporción de la población en riesgo de ictus, en parte debido a las limitaciones de los antagonistas de la vitamina $\mathrm{K}$, temor a las complicaciones de sangrado y baja percepción del riesgo trombótico, sobre todo en la población de mayor edad ${ }^{12,13}$.

El registro AFINVA (Actualidad en Flbrilación auricular No VAlvular) pretende estimar la prevalencia de fibrilación auricular en una población general no seleccionada de un Departamento de Salud, y evaluar las características y el tratamiento utilizado en la población con fibrilación auricular no valvular (FANV).

\section{Métodos}

El registro AFINVA es un estudio observacional prospectivo, diseñado con un corte transversal exploratorio y visitas de seguimiento a 1, 2 y 3 años (parte prospectiva) de una muestra de la población general de un Departamento de Salud con pacientes con fibrilación auricular, en condiciones de práctica clínica de ámbito asistencial. El Departamento atiende a 290.000 personas repartidas en 11 Zonas Básicas de Salud, de las que aparecen representadas en el registro el $91 \%$ 
mediante 20 cupos de médicos de atención primaria que suman en total 30.024 pacientes, lo que supone el $10,5 \%$ de la población atendida en el departamento. Los resultados de la parte clínica exploratoria del registro son los que se presentan en este artículo.

El estudio se realizó de forma conjunta entre cardiólogos y médicos de atención primaria del departamento de salud. Se incluyeron pacientes mayores de 18 años reclutados en el periodo comprendido entre octubre de 2014 y enero de 2015, con diagnóstico documentado de fibrilación auricular en la historia clínica electrónica de atención primaria de los cupos incluidos.

Se clasificó el tipo de fibrilación auricular según las guías vigentes ${ }^{11}$ como paroxística (remite a los 7 días o menos y es de reversión espontánea), persistente (duración mayor de 7 días, revierte con cardioversión farmacológica o eléctrica) o permanente (el ritmo de base del paciente es fibrilación auricular y es imposible o no está indicado restablecer el ritmo sinusal).

Se recopilaron datos demográficos, clínicos, pruebas complementarias disponibles más recientes y modalidades de tratamiento. El registro de variables se hizo mediante entrevista personal y consulta de la historia clínica electrónica de forma conjunta complementaria entre el médico de atención primaria y cardiología.

En los pacientes en tratamiento con antagonistas de la vitamina $\mathrm{K}$ los investigadores recogieron los valores de INR de todas las determinaciones realizadas en los 6 meses previos, con el fin de calcular el tiempo en rango terapéutico (TRT) según el método de Rosendaal et $\mathrm{al}^{14}$. No se tuvieron en cuenta los controles de anticoagulación realizados en el mes posterior a su interrupción ocasional por causa médico-quirúrgica. La prevalencia de mal control de la anticoagulación se expresa como el porcentaje de casos con TRT $<65 \%$ por el método de Rosendaal sobre el total de pacientes anticoagulados analizados.

La evaluación del riesgo de accidente cerebrovascular se determinó mediante la escala $\mathrm{CHA}_{2} \mathrm{DS}_{2} \mathrm{VASC}^{15}$, que considera cero como riesgo bajo, uno riesgo moderado y alto $\geq 2$. La escala HASBLED ${ }^{16}$ se utilizó para evaluar el riesgo de sangrado, que asigna riesgo bajo una puntuación $<3$ y alto $s i \geq 3$.

Finalmente, se incluyeron los pacientes con diagnóstico de FANV (definida por la existencia de dicha arritmia en ausencia de prótesis valvular mecánica o estenosis mitral reumática de grado al menos moderado) paroxística, permanente o persistente mayor a un mes de duración tras cardioversión fallida o no indicada, y aceptada como ritmo definitivo del paciente. Se excluyeron aquellos con fibrilación auricular valvular, fibrilación auricular menor a un mes de duración desde el diagnóstico y aquellos que no tenían información completa. El registro fue aprobado por el comité ético del hospital, y se lleva a cabo de acuerdo con los requisitos éticos expresados en la declaración de Helsinki y sus enmiendas posteriores.

\section{Análisis estadístico}

Las variables continuas se expresan como media \pm desviación estándar en caso de distribución normal, y porcentajes para variables categóricas y proporciones. Las variables continuas se compararon con una

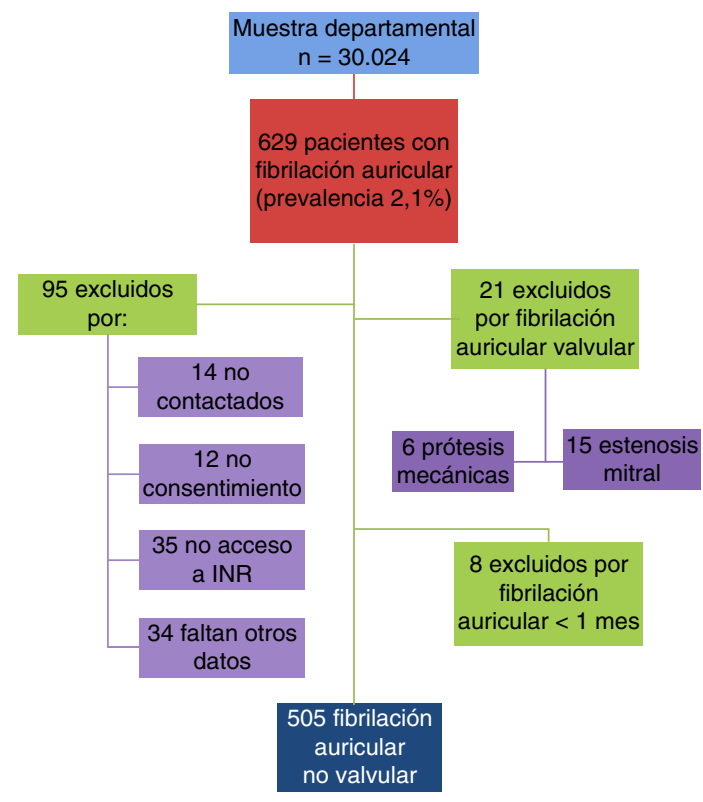

Figura 1 Diagrama de flujo de la selección de pacientes de la muestra.

INR: razón internacional normalizada.

prueba $t$, mientras que las categóricas mediante prueba de $\chi^{2}$. Los valores de $\mathrm{p}<0,05$ fueron considerados estadísticamente significativos. Los análisis se realizaron utilizando IBM SPSS Statistics ${ }^{\circledR}$ versión 19.0.0.329.

\section{Resultados}

Entre los 30.024 sujetos registrados, se detectaron 629 pacientes mayores de 18 años con fibrilación auricular, lo que representa una prevalencia del 2,1\% (fig. 1) en la población general. La presencia de fibrilación auricular aumentó con la edad (fig. 2A), alcanzando una prevalencia del 8,06\% en mayores de 65 años. Por sexos, la presentación de fibrilación auricular fue más frecuente entre los hombres hasta los 70 años, invirtiéndose esta relación en edades superiores (fig. 2B).

Tras aplicar los criterios de exclusión que aparecen en la figura 1, finalmente se incluyeron en el estudio los datos de 505 pacientes con diagnóstico de FANV. Las características basales de los pacientes con FANV aparecen en la tabla 1. La media de edad fue 77,4 \pm 10 años, el 55\% mujeres. Se destaca una proporción elevada de pacientes con hipertensión arterial (mayor en mujeres, 81,6\% vs. 74,1\%; $p<0,05$ ), hipercolesterolemia, diabetes y obesidad, así como una prevalencia de insuficiencia renal del 32,8\% (también mayor en mujeres, $36,8 \%$ vs. $28 \%$; $p<0,05$ ) definida por una tasa de filtrado glomerular CKD-EPI $<60 \mathrm{ml} / \mathrm{min} / 1,73 \mathrm{~m}^{2}$ y de cardiopatía estructural en el $32 \%$ (19,6\% isquémica y el $12,4 \%$ de otro origen). Se documentó insuficiencia cardiaca en el $29,5 \%$, con una frecuencia de disfunción sistólica ventricular izquierda (fracción de eyección del ventrículo izquierdo $<50 \%$ ) del $13,6 \%$ en los 250 casos en que se pudo disponer de examen ecocardiográfico $(49,5 \%$ de la muestra total), lo que significaría una incidencia de insuficiencia cardiaca con función sistólica preservada del 15,9\% restante. Fue llamativa la presencia de pacientes considerados 
Tabla 1 Características basales de los pacientes con fibrilación auricular no valvular (FANV). Diferencias entre FANV paroxística y persistente-permanente

\begin{tabular}{|c|c|c|c|c|}
\hline & $\begin{array}{l}\text { Total } \\
\text { (505) (\%) }\end{array}$ & $\begin{array}{l}\text { Paroxística } \\
\text { (198) (\%) }\end{array}$ & $\begin{array}{l}\text { Persistente- } \\
\text { permanente } \\
(307)(\%)\end{array}$ & $\mathrm{p}$ \\
\hline Edad media \pm SD (años) & $77,4 \pm 10$ & $73,9 \pm 11,4$ & $79,6 \pm 8,34$ & $<0,01$ \\
\hline Mujeres & $277(55)$ & $108(55)$ & $169(55)$ & 0,90 \\
\hline Fumadores & $43(8,5)$ & $19(9,5)$ & $24(7,8)$ & 0,48 \\
\hline Hipertensión arterial & $\begin{array}{l}395 \\
(78,2)\end{array}$ & $\begin{array}{l}145 \\
(73,2)\end{array}$ & $250(81,4)$ & $<0,05$ \\
\hline Diabetes mellitus & $\begin{array}{l}158 \\
(31,2)\end{array}$ & $47(23,7)$ & $111(36,1)$ & $<0,01$ \\
\hline Hipercolesterolemia & $308(61)$ & $\begin{array}{l}118 \\
(59,6)\end{array}$ & $190(61,9)$ & 0,55 \\
\hline Obesidad & $\begin{array}{l}185 \\
(36,6)\end{array}$ & $63(31,8)$ & $122(39,7)$ & 0,07 \\
\hline Ictus/AIT previo & $78(15,4)$ & $24(12,1)$ & $54(17,6)$ & 0,09 \\
\hline Alcoholismo & $10(2)$ & $3(1,5)$ & $7(2,3)$ & 0,54 \\
\hline Cardiopatía estructural & $\begin{array}{l}161 \\
(31,9)\end{array}$ & $60(30,3)$ & $101(32,9)$ & 0,54 \\
\hline Insuficiencia cardiaca & $\begin{array}{l}149 \\
(29,5)\end{array}$ & $34(17,1)$ & $115(37,4)$ & $<0,01$ \\
\hline FEVI > 50\% $(n=250)$ & $\begin{array}{l}216 \\
(86,4)\end{array}$ & $\begin{array}{l}88 / 99 \\
(89)\end{array}$ & $\begin{array}{l}128 / 151 \\
(84,7)\end{array}$ & 0,35 \\
\hline EPOC & $89(17,6)$ & $32(16,1)$ & $57(18,5)$ & 0,50 \\
\hline SAHS & $49(9,7)$ & $16(8)$ & $33(10,7)$ & 0,33 \\
\hline Frágil-dependiente & $\begin{array}{l}120 \\
(23,7)\end{array}$ & $33(16,6)$ & $88(28,6)$ & $<0,01$ \\
\hline $\mathrm{FGR}<60 \mathrm{ml} / \mathrm{m} / 1,73 \mathrm{~m}^{2}$ & $\begin{array}{l}166 \\
(32,8)\end{array}$ & $59(29,8)$ & $107(34,8)$ & 0,23 \\
\hline $\mathrm{Hb}<12 \mathrm{~g} / \mathrm{dl}$ & $111(22)$ & $45(22,7)$ & $66(21,5)$ & 0,72 \\
\hline Hipotiroidismo & $78(15,4)$ & $35(17,6)$ & $43(14)$ & 0,26 \\
\hline Hipertiroidismo & $7(1,4)$ & $3(1,5)$ & $4(1,3)$ & 0,84 \\
\hline Portador de marcapasos & $36(7,1)$ & $12(6)$ & $24(7,8)$ & 0,46 \\
\hline $\mathrm{CHA}_{2} \mathrm{DS}_{2} \mathrm{VASC} \geq 1$ & $\begin{array}{l}486 \\
(96,2)\end{array}$ & $\begin{array}{l}181 \\
(91,3)\end{array}$ & $305(99,3)$ & $<0,01$ \\
\hline HASBLED $\geq 3$ & $203(40)$ & $63(32)$ & $140(45,6)$ & $<0,01$ \\
\hline Anticoagulados & $364(72)$ & $96(48,5)$ & $268(87,3)$ & $<0,01$ \\
\hline
\end{tabular}

AIT: accidente isquémico transitorio. FEVI: fracción de eyección del ventrículo izquierdo. EPOC: enfermedad pulmonar obstructiva crónica. SAHS: síndrome apnea hipopnea del sueño. FGR: filtrado glomerular renal (CKD-EPI). Hb: hemoglobina.

frágiles o dependientes, y los afectados por alteraciones tiroideas como el hipotiroidismo, sobre todo entre las mujeres ( 30 vs. $16,2 \%$ y 21 vs. $8,7 \%$ respectivamente, ambos con $\mathrm{p}<0,01)$.

La media de la puntuación de riesgo trombótico $\mathrm{CHA}_{2} \mathrm{DS}_{2}$ VASC fue $4,07 \pm 1,8$, notablemente alta por la elevada proporción de pacientes mayores de 74 años $(66,3 \%)$, con riesgo embolígeno moderado-alto en el $96,3 \%$ de los mismos. El sexo femenino tuvo mayor puntuación media $\mathrm{CHA}_{2} \mathrm{DS}_{2} \mathrm{VASC}$ respecto a los hombres $(4,60 \pm 0,9$ vs. $3,42 \pm 1,7 ; p<0,01)$. La media de la puntuación para el riesgo de sangrado HASBLED fue $2,29 \pm 1$, con riesgo alto en el $40 \%$ de casos. En el momento de la inclusión estaban anticoagulados el $72 \%(n=364)$ de los pacientes, el $60 \%(n=304)$ con antagonistas de la vitamina $K$ y el $12 \%$ $(n=60)$ con anticoagulantes directos. Entre los pacientes tratados con antagonistas de la vitamina $\mathrm{K}$ tan solo el $53 \%$ mantenía un TRT $\geq 65 \%$ definido por INR entre $2-3$ en los últimos 6 meses (fig. 3). No hubo diferencias entre sexos en puntuación HASBLED, porcentaje de pacientes anticoagulados ni en el grado de control de la anticoagulación.

La forma de presentación más frecuente de la FANV fue la fibrilación auricular persistente-permanente $(60,8 \%)$ con una duración $\geq 6$ meses en el $96,4 \%$ de los casos, seguido de la fibrilación auricular paroxística $(39,2 \%)$. Como se observa en la figura 4, los betabloqueantes y los antagonistas del calcio no dihidropiridínicos, utilizados de forma aislada o en combinación con digoxina, fueron los fármacos más empleados en la fibrilación auricular persistente-permanente. En la fibrilación auricular paroxística los más utilizados fueron los antiarrítmicos del grupo 1 (fundamentalmente flecainida) y del grupo 3 (principalmente amiodarona). Respecto a los pacientes con fibrilación auricular persistente-permanente, aquellos con fibrilación auricular paroxística fueron de menor edad $(73,9 \pm 11,4$ vs. $79,6 \pm 8,3 p<0,001)$, anticoagulados en menor proporción $(48,4 \%$ vs. $87,3 \% p<0,001)$, 

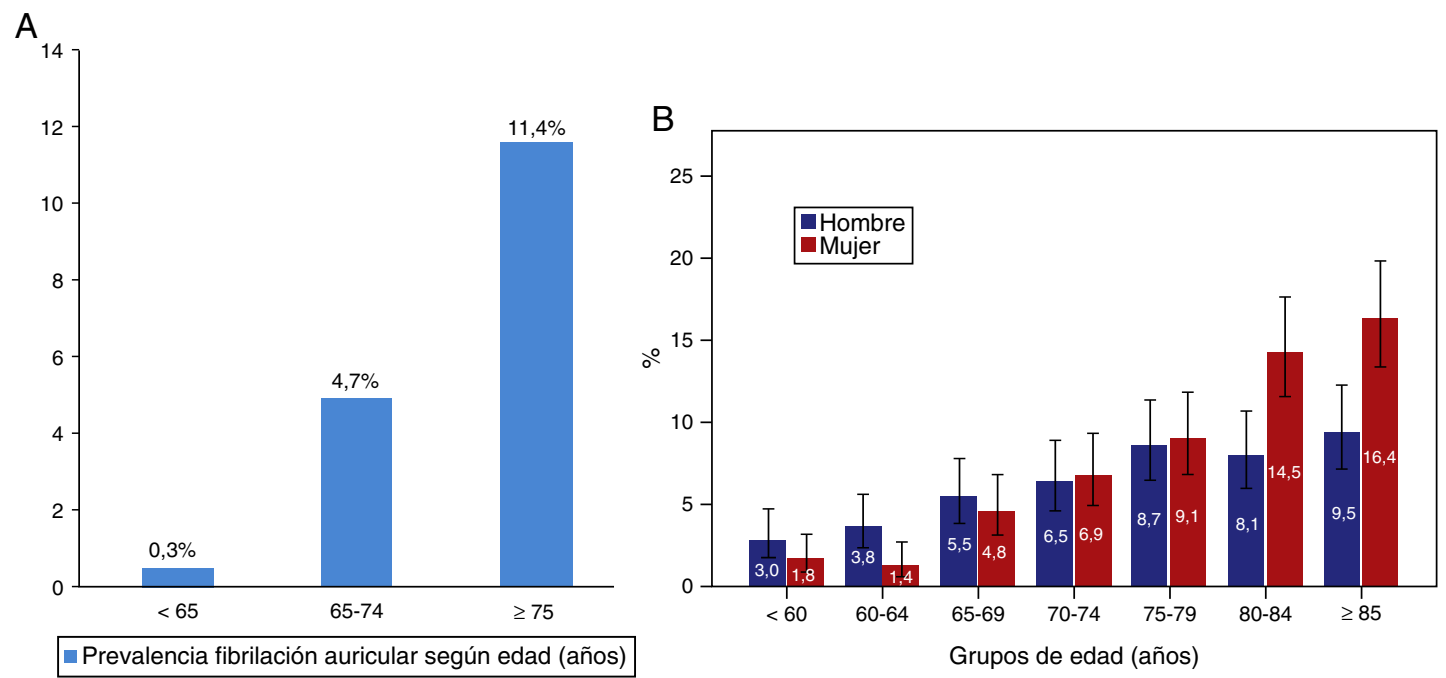

Figura 2 Prevalencia de fibrilación auricular (A) y distribución de los pacientes (B) según tramos de edad.

tuvieron menor riesgo de ictus por puntuación $\mathrm{CHA}_{2} \mathrm{DS}_{2}$ VASC $(3,5 \pm 1,9$ vs. $4,3 \pm 1,6 p=0,001)$ y de sangrado por escala HASBLED ( $2 \pm 1$ vs. $2,4 \pm 0,9 \mathrm{p}=0,001)$, así como menor proporción tanto de insuficiencia cardiaca $(17,1 \%$ vs. $37,4 \%$ $\mathrm{p}<0,001)$ como de pacientes frágiles $(16,6 \%$ vs. $28,6 \%$ $\mathrm{p}<0,01)$ (tabla 1).

\section{Discusión}

El estudio AFINVA muestra una prevalencia de fibrilación auricular del $2,1 \%$ entre 30.024 pacientes no seleccionados de atención primaria mayores de 18 años, aumentando la proporción de sujetos con fibrilación auricular al 4,7\% entre los 65-74 años y al 11,4\% en mayores de 74 años.

La mayor parte de estudios efectuados con anterioridad se han realizado en registros de pacientes de diferentes ámbitos (con otras enfermedades cardiovasculares o con limitaciones de edad), lo que ha ocasionado estimaciones muy variables, con prevalencias entre el 0,7 y el $8,5 \%{ }^{17-21}$. El estudio OFRECE ${ }^{17}$ registró una prevalencia total de fibrilación auricular del 4,4\% sobre una población española de

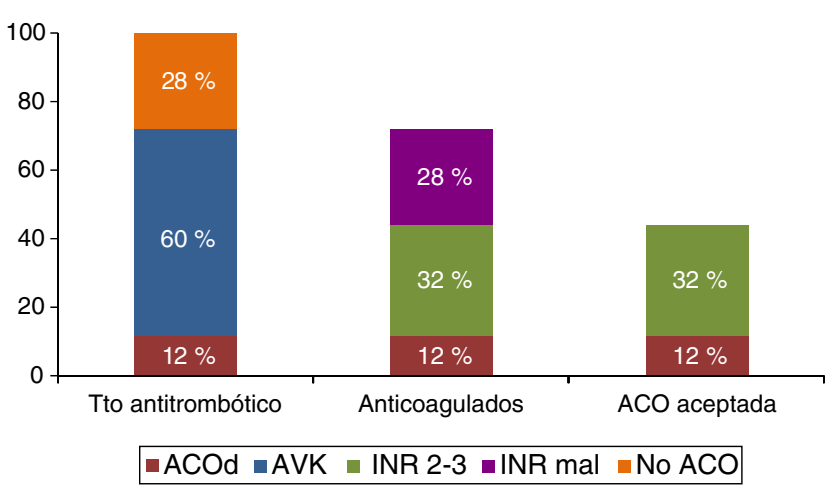

Figura 3 Tratamiento anticoagulante en pacientes con fibrilación auricular no valvular.

ACOd: anticoagulantes directos. AVK: antivitamina K. INR: razón internacional normalizada. ACO: anticoagulación oral.
40 años o más, adscrita a un médico de atención primaria. El estudio CARDIOTENS ${ }^{18}$ realizado en hipertensos, describió una prevalencia total de fibrilación auricular del $4,8 \%$, pero incluyó a pacientes que acudían a la consulta de atención primaria o de cardiología de forma consecutiva, razón por la que no puede considerarse de ámbito poblacional al suponer un sesgo de selección. El estudio REGICOR ${ }^{19}$, el único realizado en población general, apreció una prevalencia del $0,7 \%$, con la limitación importante de excluir a los pacientes mayores de 74 años que son precisamente los que mayor proporción de la arritmia presentan. Nuestro estudio representa una de las muestras contemporáneas más amplias de fibrilación auricular en la población general, hecho relevante puesto que estudios basados en población que acude a consultas hospitalarias pueden no representar la epidemiologia

\section{A Tratamiento AA y/o frenador en fibrilación auricular persistente-permanente}

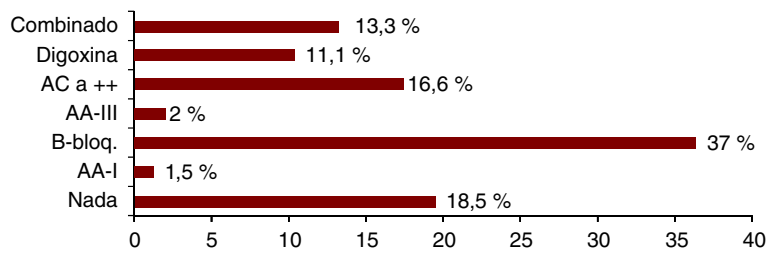

B Tratamiento AA y/o frenador en fibrilación auricular paroxística

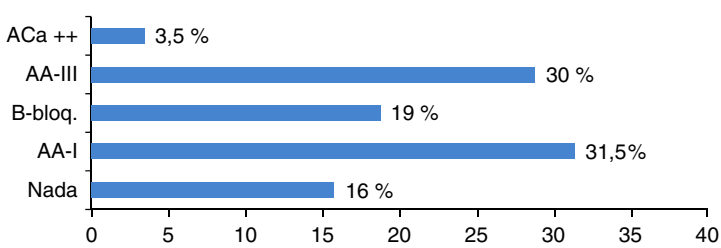

Figura 4 Fármacos antiarrítmicos o frenadores y su uso combinado en la fibrilación auricular persistente-permanente (A) y en la paroxística (B).

$\mathrm{AA}$ : antiarrítmico. FA: fibrilación auricular. $\mathrm{ACa}++$ : antagonistas del calcio no dihidropiridínicos. AA-III: antiarrítmicos del grupo 3. B-bloq: betabloqueantes. AA-ı: antiarrítmicos del grupo 1. 
clínica real, dado que una tercera parte de los sujetos con fibrilación auricular no tienen contacto hospitalario ${ }^{22}$. Se prevé que en los próximos años aumentará la prevalencia de fibrilación auricular en países occidentales debido al envejecimiento progresivo de la población, con el consiguiente aumento de los costos que ello conllevará ${ }^{23}$.

Las características clínicas de los pacientes incluidos en el actual registro son similares a las de otros estudios realizados en pacientes con FANV 2-6,17-19,21. El diseño de nuestro estudio y el registro FANTASIIA guardan algunas similitudes, si bien en este último los pacientes incluidos provenían de varios niveles asistenciales con predominio de consultas de cardiología $^{21}$. Es interesante destacar el porcentaje elevado de pacientes con FANV asistidos en atención primaria con factores de riesgo cardiovascular y enfermedad cardiovascular concomitante, principalmente insuficiencia cardiaca $(29,5 \%)$ y cardiopatía estructural $(32 \%)$, en línea con lo descrito en otros registros ${ }^{2-5,20}$.

En cuanto al riesgo tromboembólico, la puntuación media $\mathrm{CHA}_{2} \mathrm{DS}_{2}$ VASC fue mayor entre las mujeres, justificado por su mayor edad y el hecho de penalizar el sexo femenino en la puntuación de este baremo. El 96,3\% de los pacientes tenían un riesgo embolígeno intermedio-alto por puntuación $\mathrm{CHA}_{2} \mathrm{DS}_{2} \mathrm{VASC} \geq 1$, de modo que la mayoría de los pacientes con fibrilación auricular tenía indicación de anticoagulación oral. Sin embargo, más de una cuarta parte de los sujetos con $\mathrm{CHA}_{2} \mathrm{DS}_{2}$ VASC $\geq 1$ no estaba tratado con anticoagulantes. El riesgo de sangrado mediante escala HASBLED fue alto $(\geq 3)$ en el $40 \%$ de los pacientes. Son varios los estudios que muestran una infrautilización de la anticoagulación en los pacientes con fibrilación auricular ${ }^{24,25}$, lo que traduce la necesidad de mejorar la formación de los médicos acerca de las indicaciones adecuadas de la anticoagulación oral en pacientes con fibrilación auricular.

El TRT calculado mediante la fórmula de Rosendaal refleja la calidad de la anticoagulación de los pacientes en tratamiento con antagonistas de la vitamina $\mathrm{K}^{26}$, correcto si es $\geq 65 \%{ }^{27}$. Por ello durante el estudio se ha empleado este método para evaluar el grado de control en los pacientes de la muestra. El grado de control obtenido (53\% de los pacientes) coincide con lo publicado por estudios de mayor tamaño con muestras de práctica clínica, que oscilan entre el $45 \%^{28}$ y el $75,8 \%^{29}$. Lo mismo ocurre si se compara con estudios recientes, de modo que en el estudio ANFAGAL ${ }^{4}$ el $57,3 \%$ de los pacientes mostró un TRT calculado por la fórmula de Rosendaal $\geq 65 \%$, mientras fue el $52,7 \%$ de los incluidos en el estudio CALIFA ${ }^{5}$. Tanto el conjunto de pacientes en tratamiento con antagonistas de la vitamina $\mathrm{K}$ fuera de rango terapéutico, como aquellos con riesgo de sangrado alto, son quienes más claramente podrían beneficiarse de los nuevos anticoagulantes directos puesto que han mostrado un riesgo inferior que con los antagonistas de la vitamina $\mathrm{K}$ de ictus hemorrágico, hemorragia intracraneal y hemorragias mayores $^{30}$.

Un aspecto interesante es la evaluación del perfil de los pacientes en función del tipo de fibrilación auricular, persistente-permanente o paroxística. Los factores que se asociaron con la fibrilación auricular paroxística fueron menor edad, menor proporción de insuficiencia cardiaca y pacientes frágiles, y menor puntuación de riesgo en las escalas $\mathrm{CHA}_{2} \mathrm{DS}_{2}$ VASC y HASBLED. Por otra parte, al igual que en la serie de Barrios et $\mathrm{al}^{6} \mathrm{y}$ Lobos et $\mathrm{al}^{31}$, también se observaron diferencias significativas en cuanto a la anticoagulación oral y el tipo de fibrilación auricular, de tal forma que los pacientes con fibrilación auricular persistentepermanente estaban anticoagulados en mayor medida frente a los sujetos con fibrilación auricular paroxística, cuando es conocido que la indicación de anticoagulación es independiente del tipo de fibirlación auricular ${ }^{7}$.

\section{Limitaciones}

Una de las principales limitaciones del estudio deriva de su diseño transversal exploratorio, con la posibilidad de que los casos de mayor duración aparezcan representados en exceso.

Controles analíticos externos a la sanidad pública, así como la edad muy avanzada e institucionalización de algunos pacientes impidieron recabar toda su información y consecuentemente estos casos fueron excluidos.

La posibilidad de participar en el registro se ofreció a todos los médicos de atención primaria del Departamento de Salud, estando finalmente representadas en la muestra el $91 \%$ de las Zonas Básicas de Salud. Probablemente estos médicos estén más sensibilizados que el resto en esta patología, y puedan conocer y aplicar mejor las guías de anticoagulación, de modo que la realidad sería todavía más deficiente de la encontrada en esta serie.

La pirámide poblacional del Departamento de Salud objeto del estudio es superponible a la de la Comunidad Autónoma y al global del territorio nacional, así como la representación de sus variedades socioeconómicas, por lo que consideramos que la inclusión de todos los sujetos registrados con diagnóstico de fibrilación auricular en los respectivos cupos médicos de atención primaria (lo que abarca a todos los pacientes diagnosticados de fibrilación auricular en cualquier ámbito y registrados en el sistema público), tamaño y representatividad de la muestra, proporcionan fortaleza suficiente para considerarlo reflejo de la realidad. Los resultados del estudio pueden ser útiles para obtener una descripción actualizada de las características de la FANV en la práctica clínica habitual y para la planificación sanitaria.

\section{Conclusiones}

En el estudio AFINVA se observa una prevalencia de fibrilación auricular del 2,1\% en mayores de 18 años, que aumenta con la edad. Es frecuente su presentación junto a cardiopatía estructural, fundamentalmente isquémica. Los datos muestran que más de la cuarta parte de la población con FANV no está anticoagulada, sobre todo en casos de fibrilación auricular paroxística. Por otra parte, casi el $50 \%$ de los pacientes en tratamiento con antagonistas de la vitamina $\mathrm{K}$ está fuera de rango terapéutico, lo que indica que el riesgo embolígeno y hemorrágico está elevado en estos pacientes. Es necesario establecer estrategias para mejorar esta situación, como aumentar el conocimiento de los criterios de anticoagulación, optimizar la monitorización de los antagonistas de la vitamina $\mathrm{K}$ e incrementar la utilización de los nuevos anticoagulantes directos en los casos indicados. 


\section{Responsabilidades éticas}

Protección de personas y animales. Los autores declaran que los procedimientos seguidos se conformaron a las normas éticas del comité de experimentación humana responsable y de acuerdo con la Asociación Médica Mundial y la Declaración de Helsinki.

Confidencialidad de los datos. Los autores declaran que han seguido los protocolos de su centro de trabajo sobre la publicación de datos de pacientes.

Derecho a la privacidad y consentimiento informado. Los autores han obtenido el consentimiento informado de los pacientes y/o sujetos referidos en el artículo. Este documento obra en poder del autor de correspondencia.

\section{Financiación}

Los autores declaran no haber recibido ningún tipo de financiación para cualquier aspecto del trabajo presentado.

\section{Conflicto de intereses}

Los autores Vicente Mora Llabata e Ildefonso Roldán Torres declaran remuneraciones por asesoría y desarrollo de ponencias de los Laboratorios Pfizer/Bristol-Myers-Squibb, Böerhinger Ingelheim y Bayer.

\section{Anexo. Investigadores del estudio AFINVA}

Fernando Albiñana Fernández, Claudia Cabadés Rumbeu, Eliécer Casal Ardines, Silvia Casao Adriá, Daniela Dubois Marques, Héctor Hermida Revilla, Salvador Gasull Insertis, Javier González Aliaga, Patricia de Labaig Ramos, Conrado Mateu Navarro, José Simón Medina Aspas, Ana Medina Salom, Salvadora Mira Gimeno, Vicente Mora Llabata, Victoria Moreno Ballester, Miriam Moreno Prat, Fernando Navarro Ros, Julián Pacheco Arroyo, Jana Pérez Gozalbo, Carmen Pérez-Olivares Delgado, Jesús Platas Valenciano, Julio Ribelles Cebrián, Jazmín Ripoll Perelló, Vicenta María Rodrigo Salcedo, Ildefonso Roldán Torres, Maite Salavert Nadal, Juan José Sanz García, Ana Seoane Novás, María José Torres Soriano, Manuela Vicente Cañizares, Aurora Viñas Almunia.

\section{Bibliografía}

1. Davis RC, Hobbs FD, Kenkre JE, Roalfe AK, Iles R, Lip GY, Davies MK. Prevalence of atrial fibrilation in the general population and in high-risk groups: the ECHOES study. Europace. 2012;14:1553-9.

2. Kakkar AK, Mueller I, Bassand JP, Fitzmaurice DA, Goldhaber SZ, Goto S, et al. Risk profiles and antithrombotic treatment of patients newly diagnosed with atrial fibrillation at risk of stroke: Perspectives from the international, observational, prospective GARFIELD registry. PLoS ONE. 2013;8(5):e63479.

3. Hernández V, Saavedra J, Mazoteras V, López MT. Infrautilización de la anticoagulación oral en el paciente octogenario con fibrilación auricular. Perspectiva desde la práctica clínica real. Rev Colomb Cardiol. 2015;22:231-4.
4. Cinza-Sanjurjo S, Rey-Aldana D, Gestal-Pereira E, Calvo-Gómez C. Evaluación del grado de anticoagulación de pacientes con fibrilación auricular en el ámbito de atención primaria de Galicia. Estudio ANFAGAL. Rev Esp Cardiol. 2015;68(9):753-60.

5. Anguita-Sanchez M, Bertomeu-Martínez V, Cequier-Fillat A. Calidad de la anticoagulación con antagonistas de la vitamina $\mathrm{K}$ en España: prevalencia de mal control y factores asociados. Rev Esp Cardiol. 2015;68(9):761-8.

6. Barrios V, Calderón A, Escobar C, De la Figuera M. Pacientes con fibrilación auricular asistidos en consultas de atención primaria. Estudio Val-FAAP. Rev Esp Cardiol. 2012;65(1):47-53.

7. Camm AJ, Lip GY, De Caterina R, Savelieva I, Atar D, Honhloser SH, et al. 2012 focused update of the ESC Guidelines for the management of atrial fibrillation: An update of the 2010 ESC Guidelines for the management of atrial fibrillation *Developed with the special contribution of the European Heart Rhytm Association. Eur Heart J. 2012;33:2719-47.

8. Connolly SJ, Ezekowitz MD, Yusuf S, Eikelboom J, Oldgren J, Parkh A, et al. and the RE-LY Steering Committee and Investigators. Dabigatran vs warfarina in patients with atrial fibrillation. N Engl J Med. 2009;361:1139-51.

9. Patel MR, Mahaffey KW, Garg J, Pan G, Singer DE, Hacke W, et al. and the ROCKET AF Investigators. Rivaroxaban versus warfarina in nonvalvular atrial fibrillation. N Engl J Med. 2011;365: 883-91.

10. Granger CB, Alexander JH, McMurray JJ, Lopes RD, Hylek EM, Hanna M, et al. and the ARISTOTLE Committees and Investigators. Apixaban versus warfarina in patients with atrial fibrillation. N Engl J Med. 2011;365:981-92.

11. January CT, Wann LS, Alpert JS, Calkins H, Cigarroa JE, Cleveland JC, et al. 2014 AHA/ACC/HRS Guideline for the management of patients with atrial fibrillation: Executive summary. A report of the American College of Cardiology/American Heart Association Task Force on Practice Guidelines and the Heart Rhythm Society. Developed in collaboration with the Society of Thoracic Surgeons. J Am Coll Cardiol. 2014;64:2246-80.

12. Cairns JA, Connolly S, McMurtry S, Stephenson M, Talajic M. Canadian Cardiovascular Society atrial fibrillation guidelines 2010: prevention of stroke and systemic thromboembolism in atrial fibrillation and flutter. The Canadian Journal of Cardiology. 2011;27:74-90.

13. Tulner LR, Van Campen JP, Kuper IM, Gijsen GJ, Koks CH. Reasons for undertreatment with oral anticoagulants in frail geriatric outpatinets with atrial fibrillation: a prospective, descriptive study. Drugs Aging. 2010;27:39-50.

14. Rosendaal F, Cannegiester S, Van der Meer F, Briet E. A method to determine the optimal intensity of oral anticoagulant therapy. Thromb Haemost. 1993;69:236-9.

15. Lip GY, Nieuwlaat R, Pisters R, Lane DA, Crijns HJ. Defining clinical risk stratification for predicting stroke and tromboembolism in atrial fibrillation using a novel risk factor-based approach: the Euro Heart Survey on atrial fibrillation. Chest. 2010;137:263-72.

16. Pisters R, Lane DA, Nieuwlaat R, De Vos CB, Crijns HJ, Lip GY. A novel user-friendly score (HAS-BLED) to asses 1-year risk of major bleeding in patients with atrial fibrillation: the Euro Heart Survey. Chest. 2010;138:1093-100.

17. Gómez-Doblas JJ, Muñiz J, Alonso Martín JJ, Rodríguez-Roca G, Lobos JM. Prevalencia de fibrilación auricular en España. Resultados del estudio OFRECE. Rev Esp Cardiol. 2014;67(4): 259-69.

18. García-Acuña JM, González-Juanatey JR, Alegría-Ezquerra E, González-Maqueda I, Llisterri JL. La fibrilación auricular permanente en las enfermedades cardiovasculares en España. Rev Esp Cardiol. 2002;55:943-52.

19. Masia R, Sala J, Marrugat J, Pena A. Investigadores del estudio REGICOR. Prevalencia de fibrilación auricular en la provincia de Girona: el estudio REGICOR. Rev Esp Cardiol. 2001;54:1240. 
20. Cea-Calvo L, Redón J, Lozano JV, Fernández-Pérez C, MartíCanales JC, Llisterri JL, et al. investigadores del estudio PREV-ICTUS. Prevalencia de fibrilación auricular en la población española de 60 o más años de edad. Estudio PREV-ICTUS. Rev Esp Cardiol. 2007;60:616-24.

21. Roldán-Rabadán I, Anguita Sánchez M, Marín F, Quesada MA, Camacho-Siles J, Peinado R, et al. Tratamiento antiarrítmico actual de la fibrilación auricular no valvular en España. Datos del registro FANTASIIA. Rev Esp Cardiol. 2015. Disponible en: http://dx.doi.org/10.1016/j.recesp.2015.03.007.

22. Lip GY, Golding DJ, Nazir M, Beevers DG, Child DL, Fletcher RI. A survey of atrial fibrillation in general practice: the West Birmingham Atrial Fibrillation Project. Br J Gen Pract. 1997;47:285-9.

23. Ringborg A, Nieuwlaat R, Lindgren $P$, Jönsson B, Fidan D, Maggioni AP, et al. Costs of atrial fibrillation in five European countries: results from the Euro Heart Survey on atrial fibrillation. Europace. 2008;10:403-11.

24. Ogilvie IM, Newton N, Welner SA, Cowell W, Lip GY. Underuse of oral anticoagulants in atrial fibrillation: a systematic review. Am J Med. 2010;123:638-45.

25. Lip GY, Lane DA. Tratamiento anticoagulante y antiagregante plaquetario combinado en pacientes con fibrilación auricular. Rev Esp Cardiol. 2009;62:972-5.

26. Rose AJ, Hylek EM, Ozonoff A, Ash AS, Reisman JI, Berlowitz DR. Risk-adjusted percent time in therapeutic range as a quality indicator for outpatient oral anticoagulation: results of the Veterans Affairs Study to Improve Anticoagulation (VARIA). Circ Cardiovasc Qual Outcomes. 2011;4:22-9.
27. Conolly SJ, Pogue J, Eikelboom J, Flaker G, Commerford P, Franzosi MG, et al. ACTIVE W Investigators. Benefit of oral anticoagulant over antiplatelet therapy in atrial fibrillation depends on the quality international normalized ratio control achieved by centers and countries as measured by time in therapeutic range. Circulation. 2008;118:2029-37.

28. Ansell J, Hollowell J, Pengo V, Martínez Brotons F, Caro J, Drouet L. Descriptive analysis of the process and quality of oral anticoagulation management in real-life practice in patients with chronic non-valvular atrial fibrillation: the international study of anticoagulation management (ISAM). J Thromb Trombolysis. 2007;23:83-91.

29. Wieloch M, Själander A, Frykman V, Rosenquist M, Eriksson $\mathrm{N}$, Svensson PJ. Anticoagulation control in Sweden: reports of time in therapeutic range, major bleeding, and trombo-embolic complications from the national quality registry AuriculA. Eur Heart J. 2011;32:2282-9.

30. Ruff ChT, Giugliano RP, Braunwald E, Hoffman EB, Deenadayalu $N$, Ezekowitz MD, et al. Comparison of the efficacy and safety of new oral anticoagulants with warfarina in patients with atrial fibrillation: a meta-analysis of randomised trials. Lancet. 2014;383:955-62.

31. Lobos-Bejarano JM, Del Castillo-Rodríguez JC, Mena-González A, Alemán-Sánchez JJ, Cabrera de León A, Barón-Esquivias G. Investigadores del estudio FIATE (Situación actual de la Flbrilación auricular en ATención primaria en España). Características de los pacientes y abordaje terapéutico de la fibrilación auricular en atención primaria en España: Estudio FIATE. Med Clin (Barc). 2013;141:279-86. 